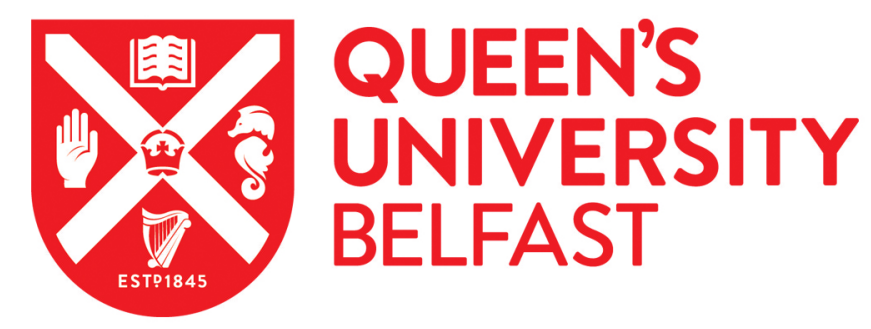

\title{
Nonclonal Emergence of Colistin Resistance Associated with Mutations in the BasRS Two-Component System in Escherichia coli Bloodstream Isolates
}

Janssen, A. B., Bartholomew, T. L., Marciszewska, N. P., Bonten, M. J. M., Willems, R. J. L., Bengoechea, J. A., \& van Schaik, W. (2020). Nonclonal Emergence of Colistin Resistance Associated with Mutations in the BasRS Two-Component System in Escherichia coli Bloodstream Isolates. mSphere, 5(2), [e00143-20]. https://doi.org/10.1128/MSPHERE.00143-20

Published in:

mSphere

Document Version:

Publisher's PDF, also known as Version of record

Queen's University Belfast - Research Portal:

Link to publication record in Queen's University Belfast Research Portal

\section{Publisher rights}

Copyright 2020 the authors.

This is an open access article published under a Creative Commons Attribution License (https://creativecommons.org/licenses/by/4.0/), which permits unrestricted use, distribution and reproduction in any medium, provided the author and source are cited.

\section{General rights}

Copyright for the publications made accessible via the Queen's University Belfast Research Portal is retained by the author(s) and / or other copyright owners and it is a condition of accessing these publications that users recognise and abide by the legal requirements associated with these rights.

\section{Take down policy}

The Research Portal is Queen's institutional repository that provides access to Queen's research output. Every effort has been made to ensure that content in the Research Portal does not infringe any person's rights, or applicable UK laws. If you discover content in the Research Portal that you believe breaches copyright or violates any law, please contact openaccess@qub.ac.uk. 


\title{
Nonclonal Emergence of Colistin Resistance Associated with Mutations in the BasRS Two-Component System in Escherichia coli Bloodstream Isolates
}

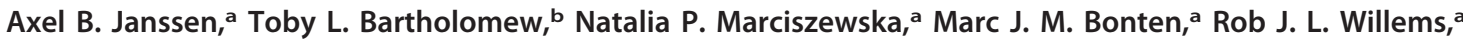 \\ Jose A. Bengoechea, ${ }^{b}$ Willem van Schaik ${ }^{a, c}$ \\ aDepartment of Medical Microbiology, University Medical Center Utrecht, Utrecht University, Utrecht, The Netherlands \\ bWellcome-Wolfson Institute for Experimental Medicine, Queen's University Belfast, Belfast, United Kingdom \\ cInstitute of Microbiology and Infection, College of Medical and Dental Sciences, University of Birmingham, Birmingham, United Kingdom
}

ABSTRACT Infections by multidrug-resistant Gram-negative bacteria are increasingly common, prompting the renewed interest in the use of colistin. Colistin specifically targets Gram-negative bacteria by interacting with the anionic lipid A moieties of lipopolysaccharides, leading to membrane destabilization and cell death. Here, we aimed to uncover the mechanisms of colistin resistance in nine colistin-resistant Escherichia coli strains and one Escherichia albertii strain. These were the only colistin-resistant strains of 1,140 bloodstream Escherichia isolates collected in a tertiary hospital over a 10-year period (2006 to 2015). Core-genome phylogenetic analysis showed that each patient was colonized by a unique strain, suggesting that colistin resistance was acquired independently in each strain. All colistin-resistant strains had lipid A that was modified with phosphoethanolamine. In addition, two $E$. coli strains had hepta-acylated lipid A species, containing an additional palmitate compared to the canonical hexa-acylated E. coli lipid A. One E. coli strain carried the mobile colistin resistance (mcr) gene mcr-1.1 on an IncX4-type plasmid. Through construction of chromosomal transgene integration mutants, we experimentally determined that mutations in basRS, encoding a two-component signal transduction system, contributed to colistin resistance in four strains. We confirmed these observations by reversing the mutations in basRS to the sequences found in reference strains, resulting in loss of colistin resistance. While the $\mathrm{mcr}$ genes have become a widely studied mechanism of colistin resistance in $E$. coli, sequence variation in basRS is another, potentially more prevalent but relatively underexplored, cause of colistin resistance in this important nosocomial pathogen.

IMPORTANCE Multidrug resistance among Gram-negative bacteria has led to the use of colistin as a last-resort drug. The cationic colistin kills Gram-negative bacteria through electrostatic interaction with the anionic lipid A moiety of lipopolysaccharides. Due to increased use in clinical and agricultural settings, colistin resistance has recently started to emerge. In this study, we used a combination of whole-genome sequence analysis and experimental validation to characterize the mechanisms through which Escherichia coli strains from bloodstream infections can develop colistin resistance. We found no evidence of direct transfer of colistin-resistant isolates between patients. The lipid A of all isolates was modified by the addition of phosphoethanolamine. In four isolates, colistin resistance was experimentally verified to be caused by mutations in the basRS genes, encoding a two-component regulatory system. Our data show that chromosomal mutations are an important cause of colistin resistance among clinical $E$. coli isolates.

KEYWORDS Escherichia coli, antibiotic resistance, colistin, two-component regulatory systems, whole-genome sequencing

Citation Janssen AB, Bartholomew TL, Marciszewska NP, Bonten MJM, Willems RJL, Bengoechea JA, van Schaik W. 2020. Nonclonal emergence of colistin resistance associated with mutations in the BasRS two-component system in Escherichia coli bloodstream isolates. mSphere 5:e00143-20. https://doi.org/10.1128/ mSphere.00143-20.

Editor Patricia A. Bradford, Antimicrobial Development Specialists, LLC

Copyright $\odot 2020$ Janssen et al. This is an open-access article distributed under the terms of the Creative Commons Attribution 4.0 International license.

Address correspondence to Willem van Schaik, w.vanschaik@bham.ac.uk.

Mutations in basRS, encoding a twocomponent signal transduction system, lead to colistin resistance in E. coli strains isolated from bloodstream infections. @axeljans @TLBartholomew @josebengoechea @MarcBonten @WvSchaik et al.

Received 13 February 2020 Accepted 20 February 2020

Published 11 March 2020 
scherichia coli is a Gram-negative opportunistic pathogen that is a common cause of bloodstream, urinary tract, and enteric infections (1). The rising prevalence of antibiotic resistance in $E$. coli, in part due to the increasing global spread of the successful multidrug-resistant clade $C$ lineage of ST131, may limit options for future treatments of infections $(2,3)$. Due to the emergence and spread of multidrug-resistant clones of E. coli and other Enterobacteriaceae, and the lack of new antibiotics targeting Gram-negative bacteria, colistin (polymyxin E) is increasingly used, despite its neuroand nephrotoxic side effects, in the treatment of clinical infections with multidrugresistant and carbapenem-resistant E. coli and other Enterobacteriaceae (4-6).

Colistin is a cationic amphipathic molecule consisting of a nonribosomal synthesized decapeptide and a lipid tail $(7,8)$. Colistin specifically targets Gram-negative bacteria by binding to the anionic phosphate groups of the lipid A moiety of lipopolysaccharides (LPS) through electrostatic interactions (7-9). Colistin destabilizes the outer membrane, but the subsequent disruption of the inner membrane ultimately leads to cell death $(9$, 10). Acquired colistin resistance has been reported in various Gram-negative bacteria that were isolated from clinical, veterinary, and environmental sources (11-13). The best-documented mechanism of colistin resistance involves the modification of lipid $A$ with cationic groups to counteract the electrostatic interactions between colistin and lipid A (9). Lipid A modifications in Enterobacteriaceae may be mediated by the acquisition of mutations in chromosomally located genes or the acquisition of a mobile genetic element carrying one of the mobile colistin resistance $(\mathrm{mcr})$ genes, which encode phosphoethanolamine transferases that catalyze the addition of a cationic phosphoethanolamine group to lipid A (14-16).

Among Enterobacteriaceae, colistin resistance has been most intensively studied in Salmonella and Klebsiella pneumoniae, in which mutations in the regulatory genes $m g r B$, pho $P Q$, and $p m r A B$ are important mechanisms leading to resistance $(15,17-19)$. In $E$. coli, however, mutations in $m g r B$ and phoPQ have not been reported to lead to colistin resistance. This may be caused by the increased rate of dephosphorylation of PmrA (BasR in E. coli) by PmrB (BasS in E. coli) in E. coli compared to that in other Enterobacteriaceae, which effectively negates the possible activating effects of mutations in phoPQ or $m g r B$, through PmrD, on the levels of phosphorylated BasR. This may explain why not all of the previously described mutations reported to lead to colistin resistance in Salmonella and Klebsiella confer resistance in E. coli (14, 20-22). In addition, phoPQ expression in $E$. coli is controlled not only by MgrB but also by the small RNA (sRNA) MicA, adding to the mechanisms controlling PhoPQ activation and making it less likely that the deletion or inactivation of $\operatorname{mgr} B$ can contribute to colistin resistance in $E$. coli $(14,23)$. This may explain why colistin resistance in clinical $E$. coli strains has only been linked to mutations in basRS (24-28), although experimental validation of the role of these mutations in colistin resistance is currently mostly lacking.

The PmrAB (BasRS) two-component system plays a crucial role in mediating the modification of LPS that leads to colistin resistance in Gram-negative bacteria $(14,17)$. Normally, this two-component system is activated by environmental stimuli, such as the presence of antimicrobial peptides or a low $\mathrm{pH}$. Activation can increase virulence and survival through evasion of the host immune system by upregulating genes associated with modification of LPS, which is the predominant immunogenic molecule of Gram-negative bacteria $(29,30)$. In E. coli, the activation of BasRS leads to increased expression of various operons, including its own. This operon also includes ept $A$, which encodes a lipid A-specific phosphoethanolamine transferase (11, 14, 31).

Relatively little is known about colistin resistance mechanisms in $E$. coli other than the acquisition of $m c r$ genes (32). Therefore, we studied a collection of colistin-resistant E. coli strains from bloodstream infections by a combination of whole-genome sequencing and matrix-assisted laser desorption ionization-time of flight (MALDI-TOF) analysis of their lipid A to identify colistin resistance mechanisms in $E$. coli. The role of mutations in basRS was investigated through the construction of chromosomal integration mutants of different basRS alleles. 
TABLE 1 Colistin-resistant Escherichia strains isolated from bloodstream infections ${ }^{a}$

\begin{tabular}{lllll}
\hline Strain & Colistin MIC $(\boldsymbol{\mu g} / \mathbf{m l})^{b}$ & MLST & Date of isolation & History of colistin use \\
\hline I1121 & 16 & 131 & 22 April 2015 & Yes; inhalation and oral \\
H2129 & 8 & 131 & 22 July 2014 & No \\
G821 & 16 & 131 & 19 March 2013 & No \\
F2745 & 4 & 73 & 2 November 2012 & No \\
E3090 & 8 & 10 & 12 November 2011 & No \\
E2372 & 4 & 59 & 25 August 2011 & No \\
E650 & 8 & 162 & 11 March 2011 & No \\
D2373 & 8 & 6901 & 20 October 2010 & Yes; oral \\
A2361 & 8 & 5268 & 3 November 2007 & No \\
Z821 & 4 & 167 & 2 April 2006 & Yes; oral \\
\hline
\end{tabular}

${ }^{a}$ Overview of colistin-resistant bloodstream isolates, including the MIC of colistin, MLST type determined through whole-genome sequencing, date of isolation, and information on the use of colistin 3 months before the isolation of the colistin-resistant isolate, and if applicable, route of administration.

${ }^{b}$ The MIC values represent the medians from three independent replicate experiments performed in triplicates.

\section{RESULTS}

Low prevalence of colistin resistance in invasive Escherichia bloodstream isolates. A total of 1,140 bloodstream isolates (collected from January 2006 to December 2015) for which species identification and automated antibiotic susceptibility testing had previously been performed, were available for this study. Twelve isolates were deemed resistant to colistin through routine diagnostic procedures. Two of those isolates were isolated from the same patient, on the same day, and were thus considered duplicates, and only one of these was included in this study. In 10 of the 11 remaining isolates, colistin resistance, defined as an MIC of $>2 \mu \mathrm{g} / \mathrm{ml}$ colistin, was confirmed through broth microdilution (Table 1). Strain A783 was a false positive for colistin resistance during automated susceptibility testing in routine diagnostic procedures and was excluded from subsequent analyses, leaving ten isolates for further investigation.

The estimated prevalence of colistin resistance in $E$. coli strains causing bloodstream infections isolated from January 2006 to December 2015 was thus determined to be $0.88 \%$. Three patients had received colistin in the 3 months before isolation of the colistin-resistant strain (Table 1). Two of these patients received colistin to treat infections, but all three patients were also administered colistin as part of selective digestive or oropharyngeal decontamination (SDD/SOD), a prophylactic antibiotic treatment widely used in Dutch intensive care units (33). The ten colistin-resistant strains were analyzed further in this study to determine their relatedness and mechanism through which they had developed colistin resistance.

Colistin resistance was independently acquired by each individual bloodstream $\boldsymbol{E}$. coli isolate. To assess the phylogenetic relationships between the colistinresistant strains, a phylogenetic tree was generated based on the genome assemblies of the colistin-resistant strains and 210 publicly available complete genome sequences (see Table S1 in the supplemental material). Based on a core genome alignment of 874 kbp, we did not observe direct transmission of colistin-resistant strains between patients (Fig. 1A). Three colistin-resistant strains (strains 11121, H2129, and G821) belonged to the globally disseminated ST131 clone, and all three were dispersed throughout the multidrug-resistant clade C of ST131 (Fig. 1A and B) $(3,34)$. This indicates that the ST131 strains in this study have independently acquired colistin resistance. Strain A2361 clustered among Escherichia albertii (Fig. 1A), although it had been typed as $E$. coli in routine diagnostic procedures.

By screening for acquired antibiotic resistance genes through ResFinder 3.2, we found that only strain E3090 carried the $\mathrm{mcr}$ gene $\mathrm{mcr}-1.1(0.086 \%$ of all bloodstream isolates) (Fig. 1C). After long-read sequencing and hybrid assembly, the $m c r-1.1$ gene in this strain appeared to be located as the sole antibiotic resistance gene on a 32.7-kbp IncX4-type plasmid. This mcr-1.1-carrying IncX4-type plasmid from E3090 shares 99\% identity to the previously reported mcr-1.1-carrying IncX4-type plasmid pMCR-1_Msc 
A
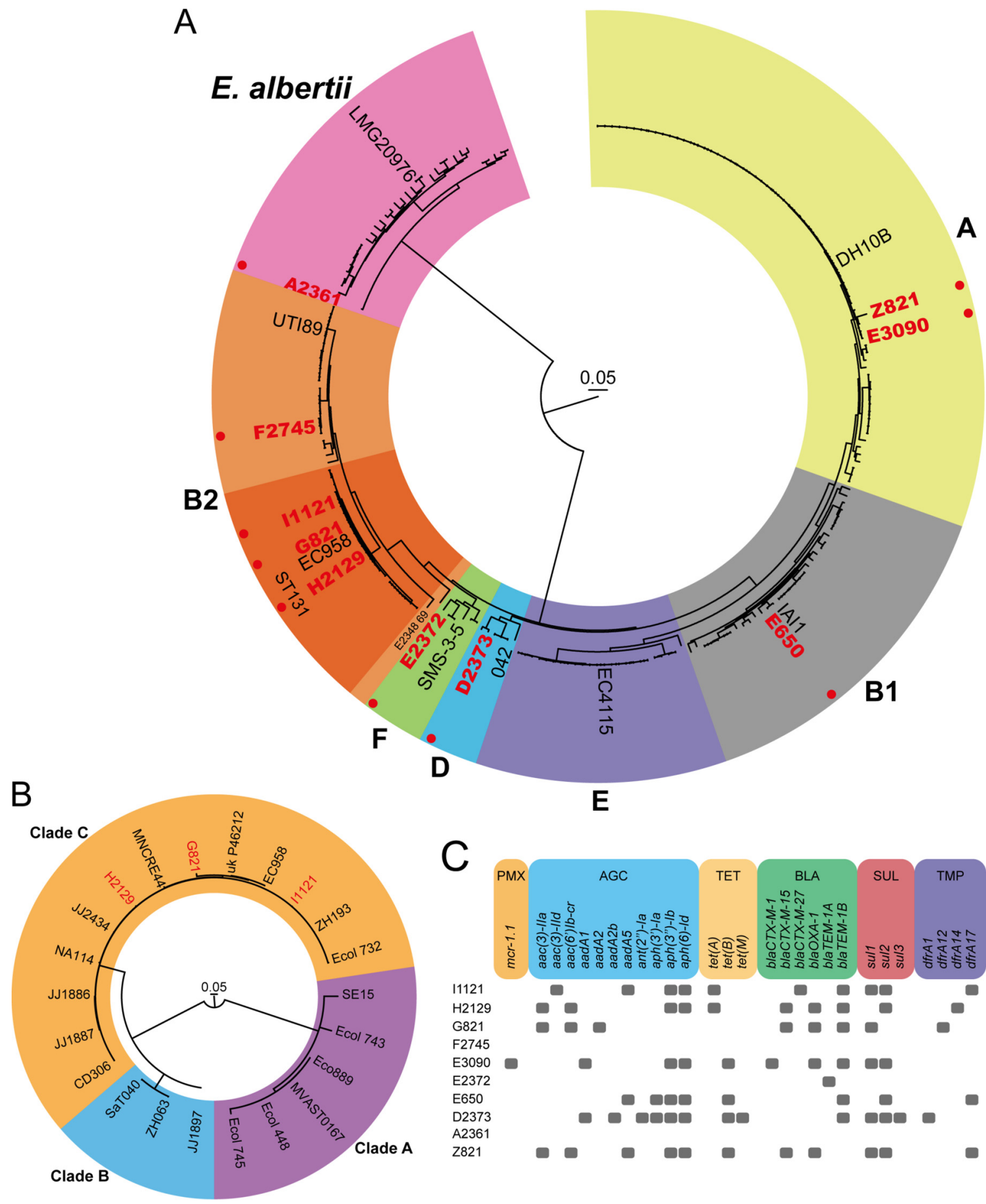

FIG 1 Colistin-resistant strains are not clonally related and carry diverse acquired antibiotic resistance genes. (A) The phylogenetic tree represents the core-genome alignment ( $874 \mathrm{kbp}$ ) of the colistin-resistant strains and 210 publicly available E. coli and E. albertii genome sequences. One representative reference strain per E. coli phylogroup is indicated (65). For E. albertii, the LMG20976 type strain is indicated (66). The different phylogroups of E. coli are indicated with colored backgrounds. Phylogroup A is yellow; B1, gray; B2, orange; D, blue; E, purple; F, green. The ST131 lineage of E. coli in phylogroup B2 is indicated by a dark orange background. The E. albertii branch is indicated by a pink background. The colistin-resistant strains characterized in this study are depicted in red and highlighted by a red filled circle. (B) The phylogenetic tree represents the core-genome alignment (3.55 Mbp) of the three colistin-resistant ST131 strains and 19 publicly available ST131 E. coli strains genome sequences. The colistin-resistant strains characterized in this study are depicted in red. Clades A, B, and C of ST131 are indicated by purple, blue, and orange backgrounds, respectively. (C) Antibiotic resistance genes in the genome sequences were detected by ResFinder 3.2 (56). Classes of antibiotic resistance genes are abbreviated as follows: PMX, polymyxin resistance; AGC, aminoglycoside resistance; TET, tetracycline resistance; BLA, beta-lactam resistance; SUL, sulfonamide resistance; TMP, trimethoprim resistance; PHE, phenicol resistance; QLN, quinolone resistance; $\mathrm{MCL}$, macrolide resistance. 
(GenBank accession MK172815.1) harbored by $E$. coli isolated from patients in Russia (35), confirming the global dissemination of this plasmid (36). In all strains studied here, a variety of acquired resistance genes was observed (Fig. 1C), reflecting the nonclonal nature of the colistin-resistant strains. The three colistin-resistant ST131 strains possessed different repertoires of acquired resistance genes, further excluding recent transmission between patients of the ST131 strains studied here. Strains F2745 and E2372 carried only one and two resistance genes, respectively, while the E. albertii strain A2361 did not possess any acquired resistance genes.

Escherichia isolates exclusively acquire colistin resistance by modification of phosphate groups of lipid A. To determine which modifications to lipid A are affecting colistin resistance in E. coli, we extracted lipid A from the clinical strains and the colistin-susceptible control E. coli strain MG1655 and subjected them to MALDI-TOF mass spectrometry. The lipid A produced by all E. coli strains showed lipid A species with a mass-to-charge ratio $(\mathrm{m} / \mathrm{z})$ of 1,797 (Fig. 2a), corresponding to the canonical unmodified $E$. coli hexa-acylated lipid A (Fig. 2b). Colistin-resistant strains showed additional lipid $A$ species at $m / z 1,921$, consistent with the addition of phosphoethanolamine $(m / z 124)$ to the hexa-acylated species. Additional species were detected in the lipid A produced by strains E650 and Z821. The species at $m / z 2,036$ indicated the addition of palmitate $(\mathrm{m} / z 239)$ to the hexa-acylated species at $m / z 1,797$, whereas the species at $m / z 2,160$ was consistent with the addition of palmitate to the hexa-acylated lipid A species containing phosphoethanolamine $(\mathrm{m} / \mathrm{z} 1,910)$.

The E. albertii strain A2361 produced lipid A distinct from that by E. coli. The species at $\mathrm{m} / \mathrm{z} 1,825$ is likely to represent a hexa-acylated species corresponding to two glucosamines, two phosphates, four $3-\mathrm{OH}-\mathrm{C}_{14}$, and two $\mathrm{C}_{14}$ (Fig. 2c). The species at $\mathrm{m} / \mathrm{z}$ 1,948 is consistent with the addition of phosphoethanolamine to the hexa-acylated species, with a further addition of palmitate to produce lipid A species at $m / z 2,187$. Species at $m / z \quad 1,868$ and $m / z \quad 2,107$ could correspond to the loss of the second phosphate group, compared to those at $m / z 1,948$ and $m / z 2,187$.

Identification of mutations in basRS as candidate mutations involved in colistin resistance. Because chromosomal mutations in basRS, but not in other regulatory systems, were previously suggested to cause colistin resistance in E. coli (24-28), we next aimed to establish the contribution of the basRS alleles in the colistin-resistant phenotype of these bloodstream isolates. Due to the multidrug-resistant nature of the clinical isolates (Fig. 1C), we were unable to generate targeted mutations in these strains. Therefore, we made chromosomal transgene insertion mutants of the different basRS alleles in the attTn7 site in the BW25113-derived $\Delta$ basRS strain BW27848 using the $\mathrm{Tn} 7$ transposon system. By making chromosomal transgenes insertions rather than using an in trans complementation method, we excluded copy number effects by plasmids and the need to use antibiotics to select for the presence of a plasmid used for in trans complementation. Since BW27848 still possesses the gene encoding the phosphoethanolamine transferase EptA, we constructed sequences that consisted of the fused sequences of the promoter region of the eptA-basR-basS operon and the basRS coding sequences in order to prevent eptA gene dosage-dependent effects. We were unable to generate the construct for strain E650, presumably due to the toxicity of the insert.

The colistin MIC determination of the generated basRS chromosomal transgene insertion mutants from strains 11121, H2129, G821, and Z821 had higher colistin MIC values than the BW27848::Tn7-empty strain, with observed MIC values $\geq 16$-fold higher than that of the BW27848::Tn7-empty strain (Table 2). As expected, the basRS allele of the $\mathrm{mcr}$-1.1-positive strain E3090 did not lead to colistin resistance. We were unable to show the contribution of basRS to colistin resistance in the additional four colistinresistant strains (F2745, E2372, D2373, and A2361) that lacked $m c r-1.1$.

Mutations in the basRS genes contribute to colistin resistance in E. coli. By construction of the chromosomal transgene insertion mutants, we identified the ability of the basRS sequences of four strains (I1121, H212, G821, and Z821) to cause colistin 

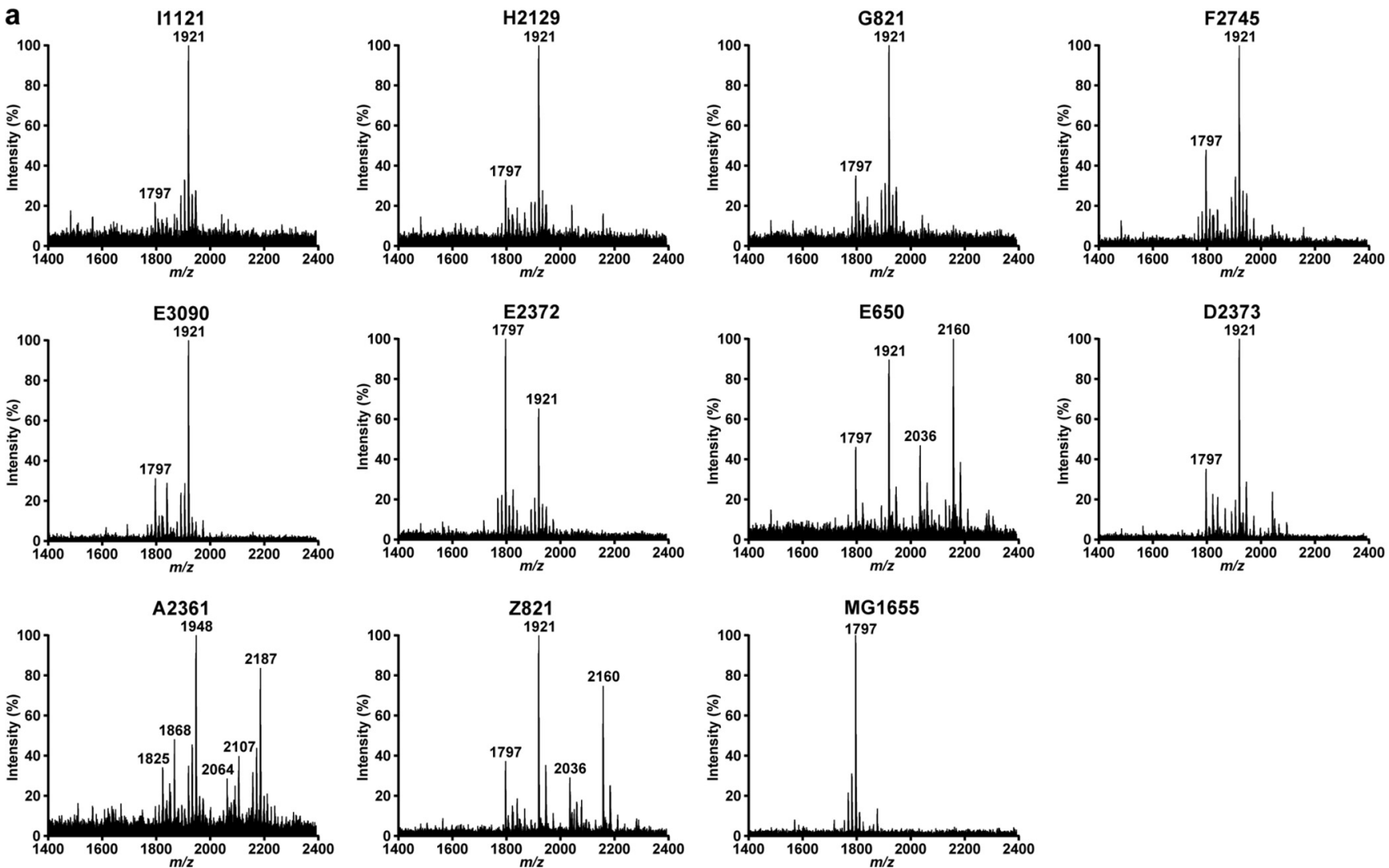

b $\quad m / z 1797$

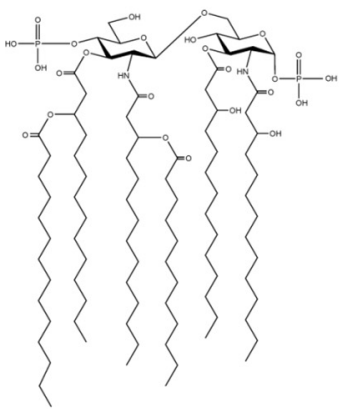

$m / z 1921$

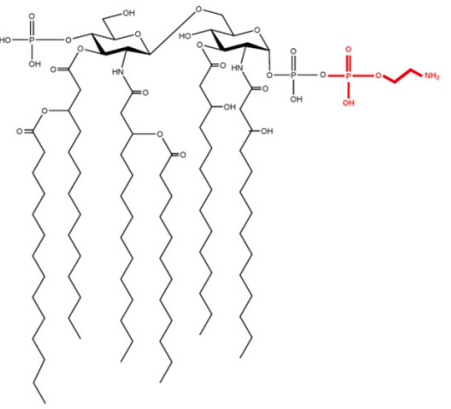

$m / z 2036$

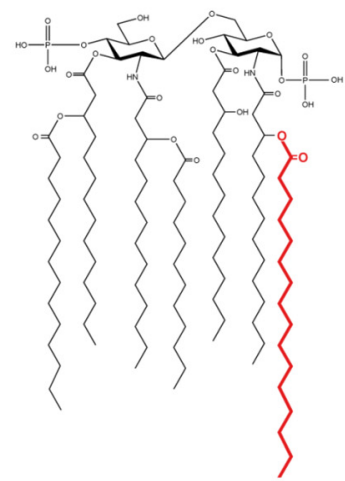

$m / z 2160$

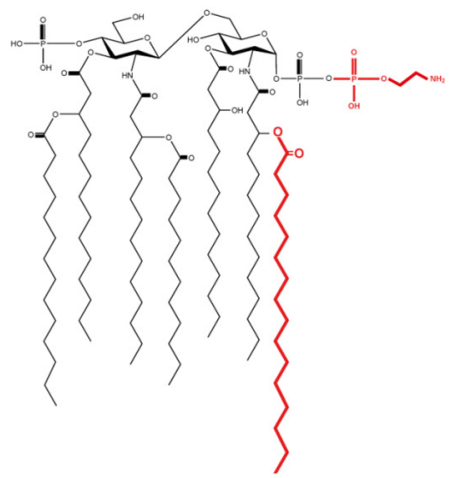

C $m / z 1825$ $m / z 1868$ $m / z 1948$ $m / z 2064$ m/z 2107 $m / z 2187$
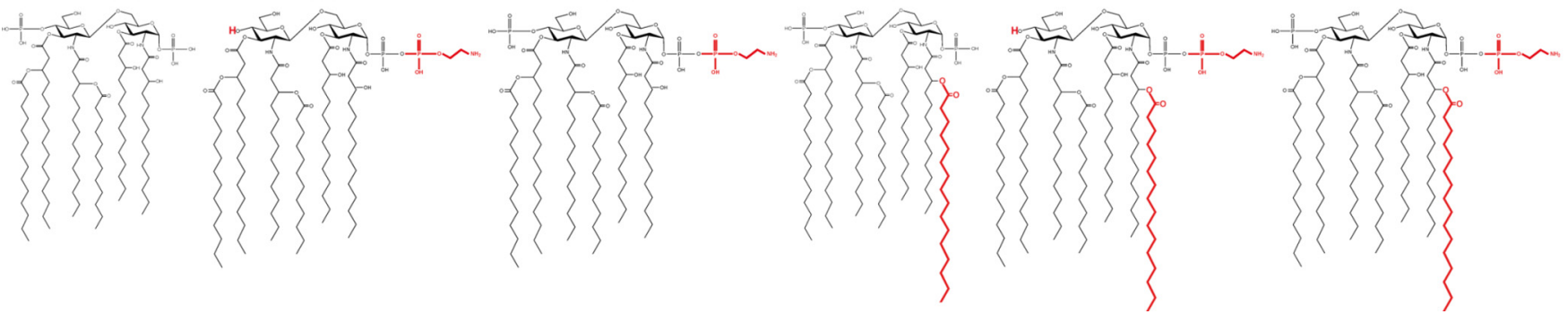

FIG 2 MALDI-TOF spectra of lipid A from colistin-resistant nosocomial Escherichia strains. (a) Negative ion MALDI-TOF mass spectrometry spectra of lipid A purified from colistin-resistant strains and colistin-susceptible MG1655. Data represent the mass to charge $(\mathrm{m} / \mathrm{z})$ ratios of each lipid A species detected and are representative of three extractions. (b) Proposed lipid A structures of the species produced by E. coli strains. (c) Proposed lipid A structures of E. albertii strain A2361. Modifications to unmodified lipid A are depicted in red. 
TABLE 2 Colistin MICs of strains generated in this study ${ }^{a}$

\begin{tabular}{ll}
\hline Strain & Colistin MIC $(\boldsymbol{\mu g} / \mathbf{m l})^{b}$ \\
\hline BW25113 & 0.25 \\
BW27848 & 0.125 \\
BW25113::Tn7 empty & 0.25 \\
BW27848::Tn7 empty & 0.125 \\
BW27848::Tn7 BW25113 & 0.125 \\
BW27848::Tn7 I1121 & 2 \\
BW27848::Tn7 I1121m & 0.25 \\
BW27848::Tn7 H2129 & 4 \\
BW27848::Tn7 H2129m & 0.25 \\
BW27848::Tn7 G821 & 4 \\
BW27848::Tn7 G821m & 0.25 \\
BW27848::Tn7 F2745 & 0.25 \\
BW27848::Tn7 E3090 & 0.125 \\
BW27848::Tn7 E2372 & 0.25 \\
BW27848::Tn7 D2373 & 0.5 \\
BW27848::Tn7 A2361 & 0.125 \\
BW27848::Tn7 Z821 & 2 \\
BW27848::Tn7 Z821m & 0.125 \\
\hline
\end{tabular}

${ }^{a} E$. coli strain BW27848 is the $\triangle b a s R S$ mutant of BW25113 (49). The bas $R S$ alleles of colistin-resistant strains from this study were inserted into the attTn7 site of BW27848. The addition of " $\mathrm{m}$ " to a strain name indicates that the construct has been modified through inverse PCR site-directed mutagenesis to reverse the mutation associated with colistin resistance.

${ }^{b}$ The values represent the medians from three independent replicate experiments performed in triplicates.

resistance in BW27848. To identify the mutations in the basRS alleles of these strains that contribute to resistance, we compared the basRS sequences of those strains causing resistance to the phylogenetically most closely related publicly available $E$. coli genome sequences used in the construction of Fig. 1A. None of these reference strains were reported to be colistin resistant or carried any of the $\mathrm{mcr}$ genes. This comparison revealed four distinct mutations: an L10R substitution in BasS in 11121, a G53S substitution in BasR in H2192, the duplication of the HAMP-domain in BasS in G821, and an A159P substitution in BasS in Z821 (Fig. 3). As expected, in the mcr-1.1-positive strain E3090, no mutations in basRS were identified.

We hypothesized that the observed mutations were impacting the normal functioning of the BasRS two-component system. To assess whether the mutations in basRS identified by comparing the basRS sequences of the clinical strains I1121, H2129, G821, and Z82, and their closest match in the set of 178 publicly available $E$. coli genome sequences (Fig. 3) were causal to the development of colistin resistance, the identified mutations were reversed through site-directed inverse PCR mutagenesis to match the

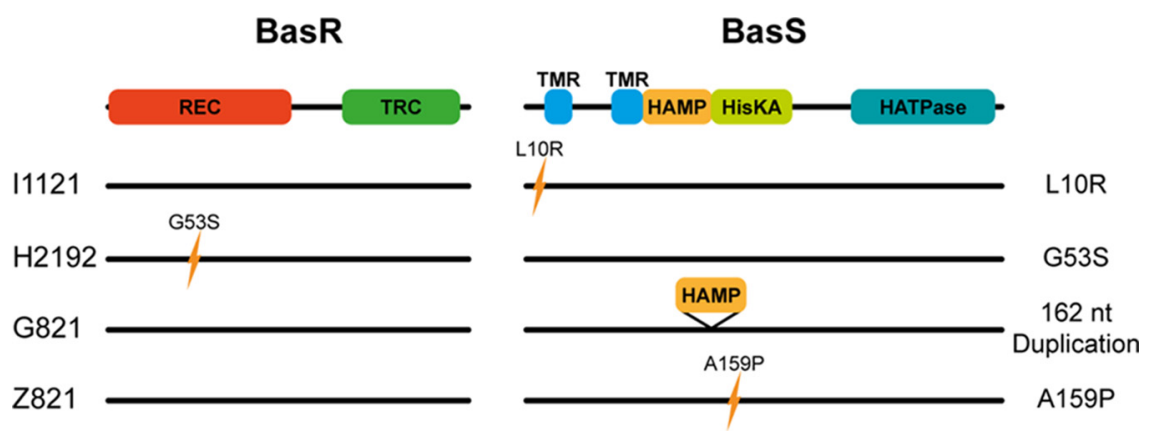

FIG 3 Conservation and prediction of functional effects of mutations in basRS. Comparison of the basRS sequences of colistin-resistant strains and publicly available genome sequences led to the identification of mutations in basRS that could have a role in colistin resistance. Domains of BasR and BasS were predicted using SMART (55). The domains are as follows: REC, CheY-homologous receiver domain; TRC, transcriptional regulatory protein, $C$ terminal (Trans_reg_c); HAMP, histidine kinases, adenylyl cyclases, methyl binding proteins, phosphatases domain; HisKA, His kinase A (phosphoacceptor) domain; HATPase, histidine kinase-like ATPases (HATPase_c). The two transmembrane regions (TMR) in BasS are highlighted in blue. 
basRS alleles of the publicly available genome sequences. The MIC values of these mutants returned to levels similar to that of the colistin-susceptible BW27848::Tn7empty strain (Table 2). These experiments support the involvement of basRS sequence variation in colistin resistance in $E$. coli.

\section{DISCUSSION}

In the present study, we set out to characterize the mechanisms through which $E$. coli bloodstream isolates can develop colistin resistance through a combination of whole-genome sequence analysis and experimental validation. We did not find evidence for transfer of colistin-resistant strains between patients, suggesting that colistin resistance was acquired independently in all cases. In seven patients, colistin-resistant strains were isolated without the patients being previously exposed to the drug. All colistin-resistant strains had LPS that was modified by the addition of phosphoethanolamine to the lipid A moiety of LPS. Resistance in one of the bloodstream isolates could be explained by the acquisition of mcr-1.1. In four other strains, we identified mutations in basRS that contribute to colistin resistance. Although colistin-susceptible strains that were isogenic to the resistant strains were not available, we were able to pinpoint the mutations in basRS leading to resistance in these strains by matching the genomic sequences of our nosocomial isolates with publicly available genomes, none of which were reported to be colistin resistant, and subsequent construction of chromosomally integrated basRS transgene alleles in the $\triangle$ basRS strain BW27848. The mechanisms of colistin resistance in the remaining five strains remain to be characterized.

Some of the mutations we experimentally link to colistin resistance in this study have previously been associated with colistin resistance or the functioning of the BasRS two-component system. In this study, we demonstrated that the amino acid change L10R in BasS (strain 11121) also confers colistin resistance. An amino acid substitution in the same position of BasS (L10P) was previously experimentally proven to cause colistin resistance in E. coli (26). The glycine in position 53 of BasR was previously reported to be altered in colistin-resistant Enterobacteriaceae $(37,38)$, including in E. coli (39). The G53S change specifically, as in isolate $\mathrm{H} 2192$, has been experimentally proven to contribute to colistin resistance in Klebsiella (previously Enterobacter) aerogenes (40, 41) and Salmonella enterica subsp. enterica serovar Typhimurium (42), and we extend those findings to E. coli here. The previously unidentified duplication of 162 nucleotides in basS (strain G821) leads to the introduction of a second HAMP domain in BasS and confers colistin resistance in the BW27848 background. The HAMP domain is widespread in bacteria and is commonly involved in signal transduction as part of twocomponent systems (43). We hypothesize that the addition of an extra HAMP domain in BasS may change signal transduction in the protein, leading to the constitutive activation of the histidine kinase domain of BasS, increased phosphorylation of BasR, and upregulated expression of eptA, ultimately resulting in the addition of phosphoethanolamine to lipid A. Finally, we demonstrate that the A159P substitution in BasS (observed in strain Z821) contributes to colistin resistance. A mutation leading to an A159V substitution was found in an in vitro evolution study in which $E$. coli was evolved toward colistin resistance (44) and in clinical colistin-resistant E. coli isolates (45), but experimental confirmation of the role of alterations in A159 in colistin resistance in $E$. coli was so far lacking. Our data suggest that the basRS alleles of three $E$ coli strains (F2745, E2372, and D2373) and the E. albertii strain A2361 do not confer resistance in the BW25113 E. coli background. Because E. albertii is phylogenetically distinct from $E$. coli, its basRS allele may not function optimally in an E. coli background, explaining the inability of the transgene insertion complementation in the basRS deletion of BW25113 E. coli strain to cause colistin resistance (46). We are unable to explain the colistin resistance mechanisms of the clinical isolates F2745, E2372, and D2373. It is likely that these strains have become resistant to colistin through other mutations that finally lead to the modification of lipid A by phosphoethanolamine.

The observed modification of lipid A with phosphoethanolamine in all isolates 
underlines the crucial role of phosphoethanolamine transferases in the ability of Escherichia to become resistant to polymyxins (14). The lipid A of three of the colistinresistant strains was also modified with palmitate, but the contribution of lipid $A$ palmitoylation to colistin resistance in clinical $E$. coli strains is currently unknown. We did not observe modifications of lipid A by 4-amino-4-deoxy-L-arabinose in the colistinresistant isolates. While this modification was shown to contribute to polymyxin $B$ resistance under low $\mathrm{Mg}^{2+}$ conditions in a laboratory isolate of $E$. coli (20), it may be rare in clinical $E$. coli isolates. Indeed, Sato et al. also exclusively found phosphoethanolamine-modified lipid A in colistin-resistant clinical E. coli isolates (24). The reliance of Escherichia on the modification of lipid A by phosphoethanolamine to acquire colistin resistance, suggests that the inhibition of this class of enzymes by blocking the conserved catalytic site (31) could be a target for future drug development and opens the possibility of combination therapy with colistin and an inhibitor of phosphoethanolamine transferase (47). With the increasing clinical issues posed by infections with multidrug-resistant Gram-negative bacteria, there is an urgent need to better understand resistance mechanisms to last-resort antibiotics such as colistin. While the discovery of the $\mathrm{mcr}$ genes has generated considerable interest in transferable colistin resistance genes, our data suggest that chromosomal mutations remain an important cause of colistin resistance among clinical isolates in the genus Escherichia.

\section{MATERIALS AND METHODS}

Ethical statement. Approval to obtain data from patient records was granted by the Medical Ethics Review Committee of the University Medical Center Utrecht, in Utrecht, The Netherlands (project numbers 16/641 and 18/472).

Colistin-resistant $E$. coli strains were isolated as part of routine diagnostic procedures. This aspect of the study did not require consent or ethical approval by an institutional review board.

Bacterial strains, growth conditions, and chemicals. Colistin-resistant $E$. coli strains from bloodstream infections were obtained retrospectively from the strain collection of the clinical microbiology laboratory of the University Medical Center Utrecht in Utrecht, The Netherlands. In initial routine diagnostic procedures, blood cultures were plated on tryptic soy agar (TSA) plates with 5\% sheep blood. Strains collected up to 2011 were identified and their antibiogram was determined using the BD Phoenix automated identification and susceptibility testing system (Becton, Dickinson, Vianen, The Netherlands). From 2011 onwards, species determination was performed by MALDI-TOF on a Bruker microflex system (Leiderdorp, The Netherlands). E. coli strain BW25113 and the BW25113-derived $\Delta$ basRS strain BW27848 from the Keio collection were obtained from the Coli Genetic Stock Center $(48,49)$. Strains were grown in lysogeny broth (LB; Oxoid, Landsmeer, The Netherlands) at $37^{\circ} \mathrm{C}$ with agitation at 300 rpm unless otherwise noted, with exception of strains containing pGRG36, which were grown at $30^{\circ} \mathrm{C}(50)$. When appropriate, kanamycin (50 mg/liter; Sigma-Aldrich, Zwijndrecht, The Netherlands) and ampicillin (100 mg/liter; Sigma-Aldrich) were used. Colistin sulfate was obtained from Duchefa Biochemie (Haarlem, The Netherlands). L-(+)-Arabinose was obtained from Sigma-Aldrich. Plasmids were purified using the GeneJET Plasmid Miniprep kit (Thermo Fisher Scientific, Landsmeer, The Netherlands). PCR products were purified from gels using GeneJET Gel Extraction and DNA Cleanup Micro kits (Thermo Fisher Scientific).

Determination of MIC. MICs to colistin were determined as previously described (51), in line with the recommendations of a joint working group of the Clinical \& Laboratory Standards Institute and the European Committee on Antimicrobial Susceptibility Testing (EUCAST) (67), using BBL Mueller-Hinton ॥ (cation-adjusted) broth (MHCAB; Becton, Dickinson), untreated Nunc 96-well round-bottom polystyrene plates (Thermo Scientific), and Breathe-Easy sealing membranes (Sigma-Aldrich). The breakpoint value of an MIC of $>2 \mu \mathrm{g} / \mathrm{ml}$ for colistin resistance in E. coli was obtained from EUCAST (http://www.eucast.org/ clinical_breakpoints/).

Genomic DNA isolation and whole-genome sequencing. Genomic DNA was isolated using the Wizard Genomic DNA purification kit (Promega, Leiden, The Netherlands) according to the manufacturer's instructions. DNA concentrations of the genomic DNA preparations were measured with the Qubit dsDNA Broad Range assay kit and the Qubit 2.0 fluorometer (Life Technologies, Bleiswijk, The Netherlands) and were all higher than $20 \mathrm{ng} / \mu \mathrm{l}$.

Sequence libraries for Illumina sequencing were prepared using the Nextera XT kit (Illumina, San Diego, CA) according to the manufacturer's instructions with $1 \mathrm{ng}$ genomic DNA as input. Libraries were sequenced on an Illumina MiSeq system with a 500-cycle ( $2 \times 250$ bp) MiSeq reagent kit v2.

For strain E3090, we performed long-read sequencing using the MinION platform (Oxford Nanopore Technologies) to fully resolve the $\mathrm{mcr}-1.1$ plasmid. MinION library preparation for barcoded twodimensional (2D) long-read sequencing was performed using the SQK-LSK208 kit (Oxford Nanopore Technologies, Oxford, United Kingdom), according to the manufacturer's instructions, with G-tube (Covaris, Woburn, MA, USA) shearing of $1 \mu \mathrm{g}$ chromosomal DNA for $2 \times 120 \mathrm{~s}$ at 1,500 $\times \mathrm{g}$. Sequencing was performed on the MinION sequencer (Oxford Nanopore Technologies) using 2D barcoded sequencing through a SpotON Flow Cell Mk I (R9.4; Oxford Nanopore Technologies). 
Genome assembly, MLST, and identification of antibiotic resistance genes. The quality of Illumina sequence data was assessed using FastQC v0.11.5 (https://github.com/s-andrews/FastQC). Raw Illumina sequencing reads were trimmed for quality using nesoni v0.115 (https://github.com/Victorian -Bioinformatics-Consortium/nesoni) using standard settings with the exception of a minimum read length of 100 nucleotides. De novo genome assembly of the trimmed Illumina short-read data was performed using SPAdes v3.6.2 with the following settings: kmers used, 21, 33, 55, 77, 99, or 127; "careful" option turned on, and cutoffs for final assemblies for a minimum contig/scaffold size of $500 \mathrm{bp}$ and minimum contig/scaffold average nucleotide coverage at 10-fold (52).

MinION sequence read data in FastQ format was extracted from Metrichor base-called raw FAST5 read-files using Poretools (53). A hybrid assembly for strain E3090 was generated with trimmed Illumina short-read data and Oxford Nanopore Technologies MinION long-read data by using SPAdes v3.6.2 with the same settings as the Illumina short-read assemblies and specifying the long-read data with the -nanopore flag.

Gene prediction and annotation was performed using Prokka (54), using standard settings. Protein domains were predicted using the SMART server (55). Multilocus sequence typing (MLST) was performed using the mlst package v2.10 (https://github.com/tseemann/mlst), using standard settings. Assembled contigs were assessed for antibiotic resistance genes using ResFinder 3.2 (56), using standard settings.

Core genome phylogenetic analysis and determination of mutations in candidate colistin resistance determinants. Genome assemblies generated in this study with Illumina data were aligned with 178 complete E. coli genomes and 32 E. albertii genomes that were available from NCBI databases on 24 June 2016 (see Table S1 in the supplemental material) using ParSNP v1.2 (57). MEGA6 was used to midpoint root and visualize the phylogenetic tree (58). We identified whether nonsynonymous mutations were present in basRS by pairwise comparison of the gene sequences of colistin-resistant isolates to their closest matching publicly available genome from the phylogenetic tree using BLAST (59). Mutations that were identified in the genome sequences were confirmed through PCR (oligonucleotide primer sequences are provided in Table S2) and subsequent Sanger sequencing of the PCR product by Macrogen (Amsterdam, The Netherlands).

Isolation and analysis of lipid A. Isolation of lipid A molecules and subsequent analysis by negative-ion MALDI-TOF mass spectrometry was performed as previously described $(19,60,61)$. Briefly, Escherichia strains were grown in LB (Oxoid), and the lipid A was purified from stationary cultures using the ammonium hydroxide-isobutyric acid method described earlier (62). Mass spectrometry analyses were performed on a Bruker autoflex speed TOF/TOF mass spectrometer in negative reflective mode with delayed extraction using as matrix equal volumes of dihydroxybenzoic acid matrix (Sigma-Aldrich) dissolved in (1:2) acetonitrile- $0.1 \%$ trifluoroacetic acid. The ion-accelerating voltage was set at $20 \mathrm{kV}$. Each spectrum was an average of 300 shots. A peptide calibration standard (Bruker) was used to calibrate the MALDI-TOF. Further calibration for lipid A analysis was performed externally using lipid A extracted from E. coli strain MG1655 grown in LB medium at $37^{\circ} \mathrm{C}$.

Construction of chromosomal basRS transgene insertions. Chromosomal transgene insertions of basRS were constructed in BW27848 by utilizing the Tn7 transposon system on the pGRG36 plasmid (50). The promoter of the eptA-basRS operon was fused to the basRS coding sequence by separate PCRs for the promoter region and the basRS amplicon, with high fidelity Phusion Green Hot Start II DNA polymerase (Thermo Fisher Scientific) using strain-specific primers (Table S2) (oligonucleotides were obtained from Integrated DNA Technologies, Leuven, Belgium). The promoter and the basRS amplicon were subsequently fused by overlap PCR. Fused PCR products were cloned into PCR-Blunt II-TOPO using the Zero Blunt TOPO PCR Cloning kit (Thermo Fisher Scientific) and subsequently subcloned into pGRG36 (50). Electrocompetent BW25113 and BW27848 E. coli cells were prepared as described previously (63) and transformed using the following settings: voltage, $1,800 \mathrm{~V}$; capacitance, $25 \mu \mathrm{F}$; resistance, $200 \Omega$; with a 0.2-cm cuvette using the Gene Pulser Xcell electroporation system (Bio-Rad Laboratories, Veenendaal, The Netherlands). Transformants were grown at $30^{\circ} \mathrm{C}$. After confirming integration of the $\mathrm{Tn} 7$ transposon at the attTn7 site by PCR (primers listed in Table S2) and Sanger sequencing (Macrogen), the pGRG36 plasmid was cleared by culturing at $37^{\circ} \mathrm{C}$.

Inverse PCR site-directed mutagenesis was performed on amplicons cloned in pCR-Blunt II-TOPO to reverse the mutations that were identified in colistin-resistant strains to the sequences of bas $R$ or bas $S$ in the closest matching publicly available genome (64). After gel purification of the amplified fragments, (hemi)methylated fragments were digested using Dpnl (New England BioLabs [NEB], Ipswich, MA, USA). Subsequently, the vector was recircularized using the Rapid DNA Ligation kit (Thermo Fisher Scientific) after phosphorylation using T4 polynucleotide kinase (NEB). The constructs were then transformed into chemically competent DH5 $\alpha$ E. coli cells (Invitrogen, Landsmeer, The Netherlands). Mutated sequences were subsequently subcloned to pGRG36 as described above.

Data availability. Sequence data have been deposited in the European Nucleotide Archive (accession number PRJEB27030).

\section{SUPPLEMENTAL MATERIAL}

Supplemental material is available online only.

TABLE S1, DOCX file, $0.1 \mathrm{MB}$.

TABLE S2, DOCX file, $0.1 \mathrm{MB}$. 


\section{ACKNOWLEDGMENTS}

We thank Eline A.M. Majoor for technical support and L. Marije Hofstra and Lidewij W. Rümke for their review of patient records. We also thank the Utrecht Sequence Facility and Ivo Renkens for their expertise in MinION Nanopore sequencing.

W.V.S. was funded through an NWO-Vidi grant (grant 917.13.357) and a Royal Society Wolfson Research Merit Award. Work in J.A.B.'s laboratory was supported by Biotechnology and Biological Sciences Research Council (BBSRC, BB/P020194/1) and Queen's University Belfast start-up. T.L.B. is the recipient of a PhD fellowship funded by the Department for Employment and Learning (Northern Ireland, UK). The funders had no role in study design, data collection and interpretation, or the decision to submit the work for publication.

A.B.J. conceived and designed experiments, performed experiments, analyzed data, and wrote the manuscript. T.L.B. and N.P.M. performed experiments and analyzed data. M.J.M.B. and R.J.L.W. wrote the manuscript. J.A.B. analyzed data and wrote the manuscript. W.V.S. conceived and designed experiments, wrote the manuscript, and supervised the study. All authors reviewed and approved the final version of the manuscript.

We declare no conflicts of interest.

\section{REFERENCES}

1. Kaper JB, Nataro JP, Mobley H. 2004. Pathogenic Escherichia coli. Nat Rev Microbiol 2:123-140. https://doi.org/10.1038/nrmicro818.

2. Kallonen T, Brodrick HJ, Harris SR, Corander J, Brown NM, Martin V, Peacock SJ, Parkhill J. 2017. Systematic longitudinal survey of invasive Escherichia coli in England demonstrates a stable population structure only transiently disturbed by the emergence of ST131. Genome Res 27:1437-1449. https://doi.org/10.1101/gr.216606.116.

3. McNally A, Kallonen T, Connor C, Abudahab K, Aanensen DM, Horner C, Peacock SJ, Parkhill J, Croucher NJ, Corander J. 2019. Diversification of colonization factors in a multidrug-resistant Escherichia coli lineage evolving under negative frequency-dependent selection. mBio 10: e00644-19. https://doi.org/10.1128/mBio.00644-19.

4. Klein EY, Van Boeckel TP, Martinez EM, Pant S, Gandra S, Levin SA, Goossens H, Laxminarayan R. 2018. Global increase and geographic convergence in antibiotic consumption between 2000 and 2015. Proc Natl Acad Sci U S A 115:E3463-E3470. https://doi.org/10.1073/pnas .1717295115 .

5. Lim LM, Ly N, Anderson D, Yang JC, Macander L, Jarkowski A, Forrest A, Bulitta JB, Tsuji BT. 2010. Resurgence of colistin: a review of resistance, toxicity, pharmacodynamics, and dosing. Pharmacotherapy 30: 1279-1291. https://doi.org/10.1592/phco.30.12.1279.

6. Nation RL, Li J. 2009. Colistin in the 21st century. Curr Opin Infect Dis 22:535-543. https://doi.org/10.1097/QCO.0b013e328332e672.

7. Domingues MM, Inácio RG, Raimundo JM, Martins M, Castanho M, Santos NC. 2012. Biophysical characterization of polymyxin B interaction with LPS aggregates and membrane model systems. Biopolymers 98: 338-344. https://doi.org/10.1002/bip.22095.

8. Landman D, Georgescu C, Martin DA, Quale J. 2008. Polymyxins revisited. Clin Microbiol Rev 21:449-465. https://doi.org/10.1128/CMR .00006-08.

9. Velkov T, Thompson PE, Nation RL, Li J. 2010. Structure-activity relationships of polymyxin antibiotics. J Med Chem 53:1898-1916. https://doi .org/10.1021/jm900999h.

10. Sabnis A, Klöckner A, Becce M, Evans LE, Furniss RCD, Mavridou DAI, Stevens MM, Edwards AM. 2018. Colistin kills bacteria by targeting lipopolysaccharide in the cytoplasmic membrane. bioRxiv 479618. https://doi.org/10.1101/479618.

11. Liu Y-Y, Wang Y, Walsh TR, Yi L-X, Zhang R, Spencer J, Doi $Y$, Tian G, Dong B, Huang X, Yu L-F, Gu D, Ren H, Chen X, Lv L, He D, Zhou H, Liang Z, Liu J-H, Shen J. 2016. Emergence of plasmid-mediated colistin resistance mechanism MCR-1 in animals and human beings in China: a microbiological and molecular biological study. Lancet Infect Dis 16:161-168. https://doi.org/10.1016/S1473-3099(15)00424-7.

12. Halaby $T$, Kucukkose $E$, Janssen $A B$, Rogers MRC, Doorduijn DJ, van der Zanden AGM, AI Naiemi N, Vandenbroucke-Grauls C, van Schaik W. 2016. Genomic characterization of colistin heteroresistance in Klebsiella pneu- moniae during a nosocomial outbreak. Antimicrob Agents Chemother 60:6837-6843. https://doi.org/10.1128/AAC.01344-16.

13. Islam A, Rahman Z, Monira S, Rahman MA, Camilli A, George CM, Ahmed N, Alam M. 2017. Colistin resistant Escherichia coli carrying $\mathrm{mcr}-1$ in urban sludge samples: Dhaka, Bangladesh. Gut Pathog 9:77. https://doi .org/10.1186/s13099-017-0227-4.

14. Baron S, Hadjadj L, Rolain J-M, Olaitan AO. 2016. Molecular mechanisms of polymyxin resistance: knowns and unknowns. Int J Antimicrob Agents 48:583-591. https://doi.org/10.1016/j.ijantimicag.2016.06.023.

15. Poirel L, Jayol A, Nordmann P. 2017. Polymyxins: antibacterial activity, susceptibility testing, and resistance mechanisms encoded by plasmids or chromosomes. Clin Microbiol Rev 30:557-596. https://doi.org/10 .1128/CMR.00064-16.

16. Zhang H, Srinivas S, Xu Y, Wei W, Feng Y. 2019. Genetic and biochemical mechanisms for bacterial lipid A modifiers associated with polymyxin resistance. Trends Biochem Sci 44:973-988. https://doi.org/10.1016/j.tibs .2019.06.002.

17. Falagas ME, Rafailidis PI, Matthaiou DK. 2010. Resistance to polymyxins: mechanisms, frequency and treatment options. Drug Resist Updat 13: 132-138. https://doi.org/10.1016/j.drup.2010.05.002.

18. Cannatelli A, D'Andrea MM, Giani T, Di Pilato V, Arena F, Ambretti S, Gaibani P, Rossolini GM. 2013. In vivo emergence of colistin resistance in Klebsiella pneumoniae producing KPC-type carbapenemases mediated by insertional inactivation of the $\mathrm{PhoQ} / \mathrm{PhoP} \mathrm{mgrB}$ regulator. Antimicrob Agents Chemother 57:5521-5526. https://doi.org/10.1128/ AAC.01480-13.

19. Kidd TJ, Mills G, Sá-Pessoa J, Dumigan A, Frank CG, Insua JL, Ingram R, Hobley L, Bengoechea JA. 2017. A Klebsiella pneumoniae antibiotic resistance mechanism that subdues host defences and promotes virulence. EMBO Mol Med 9:430-447. https://doi.org/10.15252/emmm .201607336.

20. Rubin EJ, Herrera CM, Crofts AA, Trent MS. 2015. PmrD is required for modifications to Escherichia coli endotoxin that promote antimicrobial resistance. Antimicrob Agents Chemother 59:2051-2061. https://doi .org/10.1128/AAC.05052-14.

21. Winfield MD, Groisman EA. 2004. Phenotypic differences between Salmonella and Escherichia coli resulting from the disparate regulation of homologous genes. Proc Natl Acad Sci U S A 101:17162-17167. https:// doi.org/10.1073/pnas.0406038101.

22. Chen HD, Jewett MW, Groisman EA. 2011. Ancestral genes can control the ability of horizontally acquired loci to confer new traits. PLoS Genet 7:e1002184. https://doi.org/10.1371/journal.pgen.1002184.

23. Coornaert A, Lu A, Mandin P, Springer M, Gottesman S, Guillier M. 2010. MicA sRNA links the PhoP regulon to cell envelope stress. Mol Microbiol 76:467-479. https://doi.org/10.1111/j.1365-2958.2010.07115.x.

24. Sato $T$, Shiraishi T, Hiyama $Y$, Honda H, Shinagawa $M$, Usui M, Kuronuma K, Masumori N, Takahashi S, Tamura Y, Yokota SJ. 2018. Contribution of 
novel amino alterations in PmrA or PmrB to colistin resistance in $\mathrm{mcr}$ negative Escherichia coli clinical isolates, including major multidrugresistant lineages O25b:H4-ST131-H30Rx and non-x. Antimicrob Agents Chemother 62:e00864-18. https://doi.org/10.1128/AAC.00864-18.

25. Froelich JM, Tran K, Wall D. 2006. A pmrA constitutive mutant sensitizes Escherichia coli to deoxycholic acid. J Bacteriol 188:1180-1183. https:// doi.org/10.1128/JB.188.3.1180-1183.2006.

26. Cannatelli A, Giani T, Aiezza N, Di Pilato V, Principe L, Luzzaro F, Galeotti $\mathrm{CL}$, Rossolini GM. 2017. An allelic variant of the PmrB sensor kinase responsible for colistin resistance in an Escherichia coli strain of clinical origin. Sci Rep 7:5071. https://doi.org/10.1038/s41598-017-05167-6.

27. Delannoy S, Le Devendec L, Jouy E, Fach P, Drider D, Kempf I. 2017. Characterization of colistin-resistant Escherichia coli isolated from diseased pigs in France. Front Microbiol 8:2278. https://doi.org/10.3389/ fmicb.2017.02278.

28. Bourrel AS, Poirel L, Royer G, Darty M, Vuillemin X, Kieffer N, Clermont O, Denamur E, Nordmann P, Decousser J-W, IAME Resistance Group. 2019. Colistin resistance in Parisian inpatient faecal Escherichia coli as the result of two distinct evolutionary pathways. J Antimicrob Chemother 74: 1521-1530. https://doi.org/10.1093/jac/dkz090.

29. Park BS, Song DH, Kim HM, Choi B-S, Lee H, Lee J-O. 2009. The structural basis of lipopolysaccharide recognition by the TLR4-MD-2 complex. Nature 458:1191-1196. https://doi.org/10.1038/nature07830.

30. Maeshima N, Fernandez RC. 2013. Recognition of lipid A variants by the TLR4-MD-2 receptor complex. Front Cell Infect Microbiol 3:3. https://doi .org/10.3389/fcimb.2013.00003.

31. Xu Y, Wei W, Lei S, Lin J, Srinivas S, Feng Y. 2018. An evolutionarily conserved mechanism for intrinsic and transferable polymyxin resistance. mBio 9:e02317-17. https://doi.org/10.1128/mBio.02317-17.

32. Luo Q, Yu W, Zhou K, Guo L, Shen P, Lu H, Huang C, Xu H, Xu S, Xiao Y, Li L. 2017. Molecular epidemiology and colistin resistant mechanism of mcr-positive and mcr-negative clinical isolated Escherichia coli. Front Microbiol 8:2262. https://doi.org/10.3389/fmicb.2017.02262.

33. Oostdijk EAN, Kesecioglu J, Schultz MJ, Visser CE, de Jonge E, van Essen EHR, Bernards AT, Purmer I, Brimicombe R, Bergmans D, van Tiel F, Bosch $F H$, Mascini E, van Griethuysen A, Bindels A, Jansz A, van Steveninck FAL, van der Zwet WC, Fijen JW, Thijsen S, de Jong R, Oudbier J, Raben A, van der Vorm E, Koeman M, Rothbarth P, Rijkeboer A, Gruteke P, Hart-Sweet $H$, Peerbooms $P$, Winsser LJ, van Elsacker-Niele A-MW, Demmendaal K, Brandenburg A, de Smet AMGA, Bonten MJM. 2014. Effects of decontamination of the oropharynx and intestinal tract on antibiotic resistance in ICUs a randomized clinical trial. JAMA 312:1429-1437. https://doi.org/ 10.1001/jama.2014.7247.

34. Ben Zakour NL, Alsheikh-Hussain AS, Ashcroft MM, Khanh Nhu NT, Roberts LW, Stanton-Cook M, Schembri MA, Beatson SA. 2016. Sequential acquisition of virulence and fluoroquinolone resistance has shaped the evolution of Escherichia coli ST131. mBio 7:e00347-16. https://doi .org/10.1128/mBio.00347-16.

35. Ageevets V, Lazareva I, Mrugova T, Gostev V, Lobzin Y, Sidorenko S. 2019. IncX4 plasmids harbouring $\mathrm{mcr}-1$ genes: further dissemination. J Glob Antimicrob Resist 18:166-167. https://doi.org/10.1016/j.jgar.2019.07.002.

36. Wang $R$, van Dorp L, Shaw LP, Bradley P, Wang Q, Wang X, Jin L, Zhang Q, Liu Y, Rieux A, Dorai-Schneiders T, Weinert LA, Iqbal Z, Didelot $X$, Wang H, Balloux F. 2018. The global distribution and spread of the mobilized colistin resistance gene $m c r-1$. Nat Commun 9:1179. https:// doi.org/10.1038/s41467-018-03205-z.

37. Nordmann P, Jayol A, Poirel L. 2016. Rapid detection of polymyxin resistance in Enterobacteriaceae. Emerg Infect Dis 22:1038-1043. https:// doi.org/10.3201/eid2206.151840.

38. Olaitan AO, Diene SM, Kempf M, Berrazeg M, Bakour S, Gupta SK, Thongmalayvong B, Akkhavong K, Somphavong S, Paboriboune P, Chaisiri K, Komalamisra C, Adelowo OO, Fagade OE, Banjo OA, Oke AJ, Adler A, Assous MV, Morand S, Raoult D, Rolain JM. 2014. Worldwide emergence of colistin resistance in Klebsiella pneumoniae from healthy humans and patients in Lao PDR, Thailand, Israel, Nigeria and France owing to inactivation of the $\mathrm{PhoP} / \mathrm{PhoQ}$ regulator $\mathrm{mgrB}$ : an epidemiological and molecular study. Int J Antimicrob Agents 44:500-507. https://doi .org/10.1016/j.ijantimicag.2014.07.020.

39. Trent MS, Ribeiro AA, Doerrler WT, Lin S, Cotter RJ, Raetz C. 2001. Accumulation of a polyisoprene-linked amino sugar in polymyxinresistant Salmonella typhimurium and Escherichia coli: structural characterization and transfer to lipid $A$ in the periplasm. J Biol Chem 276: 43132-43144. https://doi.org/10.1074/jbc.M106962200.

40. Diene SM, Merhej V, Henry M, El Filali A, Roux V, Robert C, Azza S, Gavory
F, Barbe V, La Scola B, Raoult D, Rolain JM. 2013. The rhizome of the multidrug-resistant Enterobacter aerogenes genome reveals how new "killer bugs" are created because of a sympatric lifestyle. Mol Biol Evol 30:369-383. https://doi.org/10.1093/molbev/mss236.

41. Tindall BJ, Sutton G, Garrity GM. 2017. Enterobacter aerogenes Hormaeche and Edwards 1960 (approved lists 1980) and Klebsiella mobilis Bascomb et al. 1971 (approved lists 1980) share the same nomenclatural type (ATCC 13048) on the approved lists and are homotypic synonyms, with consequences for the name Klebsiella mobilis Basacomb et al. 1971 (approved Lists 1980). Int J Syst Evol Microbiol 67:502-504. https://doi .org/10.1099/ijsem.0.001572.

42. Sun S, Negrea A, Rhen M, Andersson DI. 2009. Genetic analysis of colistin resistance in Salmonella enterica serovar Typhimurium. Antimicrob Agents Chemother 53:2298-2305. https://doi.org/10.1128/AAC.01016-08.

43. Schultz JE, Kanchan K, Ziegler M. 2015. Intraprotein signal transduction by HAMP domains: a balancing act. Int J Med Microbiol 305:243-251. https://doi.org/10.1016/j.ijmm.2014.12.007.

44. Lee J-Y, Choi M-J, Choi HJ, Ko KS. 2016. Preservation of acquired colistin resistance in Gram-negative bacteria. Antimicrob Agents Chemother 60:609-612. https://doi.org/10.1128/AAC.01574-15.

45. Olaitan AO, Morand S, Rolain JM. 2016. Emergence of colistin-resistant bacteria in humans without colistin usage: a new worry and cause for vigilance. Int J Antimicrob Agents 47:1-3. https://doi.org/10.1016/j ijantimicag.2015.11.009.

46. van der Putten BCL, Matamoros S, COMBAT Consortium, Schultsz C. 2019. Genomic evidence for revising the Escherichia genus and description of Escherichia ruysiae sp. nov. bioRxiv https://www.biorxiv.org/ content/10.1101/781724v2.

47. Son SJ, Huang R, Squire CJ, Leung I. 2019. MCR-1: a promising target for structure-based design of inhibitors to tackle polymyxin resistance. Drug Discov Today 24:206-216. https://doi.org/10.1016/j.drudis.2018.07.004.

48. Baba T, Ara T, Hasegawa M, Takai Y, Okumura Y, Baba M, Datsenko KA, Tomita M, Wanner BL, Mori H. 2006. Construction of Escherichia coli K-12 in-frame, single-gene knockout mutants: the Keio collection. Mol Syst Biol 2:2006.0008. https://doi.org/10.1038/msb4100050.

49. Zhou L, Lei X, Bochner BR, Wanner BL. 2003. Phenotype microarray analysis of Escherichia coli K-12 mutants with deletions of all twocomponent systems. J Bacteriol 185:4956-4972. https://doi.org/10 .1128/jb.185.16.4956-4972.2003.

50. McKenzie GJ, Craig NL. 2006. Fast, easy and efficient: site-specific insertion of transgenes into enterobacterial chromosomes using Tn7 without need for selection of the insertion event. BMC Microbiol 6:39. https:// doi.org/10.1186/1471-2180-6-39.

51. Andrews JM. 2001. Determination of minimum inhibitory concentrations. J Antimicrob Chemother 48:5-16. https://doi.org/10.1093/jac/48 .suppl_1.5.

52. Bankevich A, Nurk S, Antipov D, Gurevich AA, Dvorkin M, Kulikov AS, Lesin VM, Nikolenko SI, Pham S, Prjibelski AD, Pyshkin AV, Sirotkin AV, Vyahhi N, Tesler G, Alekseyev MA, Pevzner PA. 2012. SPAdes: a new genome assembly algorithm and its applications to single-cell sequencing. J Comput Biol 19:455-477. https://doi.org/10.1089/cmb.2012.0021.

53. Loman NJ, Quinlan AR. 2014. Poretools: a toolkit for analyzing nanopore sequence data. Bioinformatics 30:3399-3401. https://doi.org/10.1093/ bioinformatics/btu555.

54. Seemann T. 2014. Prokka: rapid prokaryotic genome annotation. Bioinformatics 30:2068-2069. https://doi.org/10.1093/bioinformatics/btu153.

55. Letunic I, Bork P. 2018. 20 years of the SMART protein domain annotation resource. Nucleic Acids Res 46:D493-D496. https://doi.org/10.1093/ nar/gkx922.

56. Zankari E, Hasman H, Cosentino S, Vestergaard M, Rasmussen S, Lund O, Aarestrup FM, Larsen MV. 2012. Identification of acquired antimicrobial resistance genes. J Antimicrob Chemother 67:2640-2644. https://doi .org/10.1093/jac/dks261.

57. Treangen TJ, Ondov BD, Koren S, Phillippy AM. 2014. The Harvest suite for rapid core-genome alignment and visualization of thousands of intraspecific microbial genomes. Genome Biol 15:524. https://doi.org/10 $.1186 / \mathrm{s} 13059-014-0524-x$.

58. Tamura K, Stecher G, Peterson D, Filipski A, Kumar S. 2013. MEGA6: Molecular Evolutionary Genetics Analysis version 6.0. Mol Biol Evol 30:2725-2729. https://doi.org/10.1093/molbev/mst197.

59. Altschul SF, Gish W, Miller W, Myers EW, Lipman DJ. 1990. Basic Local Alignment Search Tool. J Mol Biol 215:403-410. https://doi.org/10.1016/ S0022-2836(05)80360-2.

60. Llobet E, Martínez-Moliner V, Moranta D, Dahlström KM, Regueiro V, 
Tomás A, Cano V, Pérez-Gutiérrez C, Frank CG, Fernández-Carrasco H, Insua JL, Salminen TA, Garmendia J, Bengoechea JA. 2015. Deciphering tissue-induced Klebsiella pneumoniae lipid A structure. Proc Natl Acad Sci U S A 112:E6369-E6378. https://doi.org/10.1073/pnas.1508820112.

61. Llobet E, Campos MA, Giménez P, Moranta D, Bengoechea JA. 2011. Analysis of the networks controlling the antimicrobial-peptidedependent induction of Klebsiella pneumoniae virulence factors. Infect Immun 79:3718-3732. https://doi.org/10.1128/IAl.05226-11.

62. El Hamidi A, Tirsoaga A, Novikov A, Hussein A, Caroff M. 2005. Microextraction of bacterial lipid A: easy and rapid method for mass spectrometric characterization. J Lipid Res 46:1773-1778. https://doi.org/10 .1194/jlr.D500014-JLR200.

63. Sharma RC, Schimke RT. 1996. Preparation of electro-competent E. coli using salt-free growth medium. Biotechniques 20:42-44. https://doi.org/ 10.2144/96201bm08.

64. Silva D, Santos G, Barroca M, Collins T. 2017. Inverse PCR for point mutation introduction, $\mathrm{p}$ 87-100. Methods in molecular biology. Humana Press Inc., Totowa, NJ.

65. McNally A, Cheng L, Harris SR, Corander J. 2013. The evolutionary path to extraintestinal pathogenic, drug-resistant Escherichia coli is marked by drastic reduction in detectable recombination within the core genome. Genome Biol Evol 5:699-710. https://doi.org/10.1093/ gbe/evt038.

66. Huys G, Cnockaert M, Janda JM, Swings J. 2003. Escherichia albertii sp. nov., a diarrhoeagenic species isolated from stool specimens of Bangladeshi children. Int J Syst Evol Microbiol 53:807-810. https://doi.org/10 .1099/ijs.0.02475-0.

67. Joint CLSI-EUCAST Polymyxin Breakpoints Working Group. 2016. Recommendations for MIC determination of colistin (polymyxin E). EUCAST, Växjö, Sweden. http://www.eucast.org/fileadmin/src/media/PDFs/ EUCAST_files/General_documents/Recommendations_for_MIC determination_of_colistin_March_2016.pdf 